\title{
A THREE-DIMENSIONAL MODEL STUDY OF CHEMISTRY IN THE LOWER STRATOSPHERE
}

\author{
D. Lary, G. Carver and J. A. Pyle \\ Department of Chemistry, University of Cambridge, Lensfield Road, Cambridge, \\ $C B 21 E W, U . K$.
}

\begin{abstract}
This paper presents first results of an integration with a new three-dimensional atmospheric photochemical model. A fourteen day integration, commencing with the meteorological analysis of $15 \mathrm{Jan}$ 1987, is studied. The $\mathrm{NO}_{2}$ column shows a high-latitude feature similar to the "Noxon cliff". Preliminary analysis of this is presented.
\end{abstract}

\section{INTRODUCTION}

Modelling studies of atmospheric chemistry have advanced considerably in recent years. In the early 1970's simple one-dimensional (height only) models were the principal tools of the atmospheric chemist. In the late 1970's, and increasingly during the 1980's, two-dimensional model studies were carried out. These attempted to include the important details of the meridional transport of trace species and, in some cases, to include details of the feedback between ozone concentration, radiative heating and the mean circulation. While these models have had their successes it is clear that many atmospheric phenomena cannot be explained satisfactorily in a two-dimensional framework. On the other hand, the constraints of available computing power have limited the number of studies of atmospheric chemistry using three-dimensional models. In recent years a number of groups have developed such models $/ 1,2,3,4 /$ and their use will certainly grow, driven by both the ever increasing power of computers and the availability of three-dimensional data sets from satellite instruments.

This paper presents a description of some chemical studies made using a spectral general circulation model. The initial model was made available by the European Centre for Medium Range Forecasts (ECMWF) and has been modified for various research activities by researchers of the UK Universities Global Atmospheric Modelling Project (UGAMP) involving the universities of Reading, Cambridge, Edinburgh, Oxford, Imperial College London and the Rutherford Appleton Laboratory. The studies described here use the chemical schemes developed by the Atmospheric Chemistry Modelling Group at the Department of Chemistry in Cambridge.

Some preliminary results of a fourteen day integration are presented. We have studied in particular the behaviour of the oxides of nitrogen during a simulated stratospheric warming. Low values of the $\mathrm{NO}_{2}$ column are found in the model in high latitudes, a feature similar to that first reported by Noxon 15,6,7/. Reasons for the appearance of the feature are presented.

\section{THE UGAMP MODEL}

The UGAMP general circulation model (UGCM) is based on the forecast model of the ECMWF. It uses the spectral method in the horizontal and a finite difference scheme in the vertical. The model resolution can be altered as required. The resolution used for this study is a triangular 
truncation at wavenumber 21 , corresponding approximately to a $6^{\circ} \times 6^{\circ}$ grid, with 19 levels in the vertical. The top model level is at $10 \mathrm{mb}$. The vertical coordinate is the hybrid sigma coordinate system of Simmons and Burridge /8/. The model also contains a sophisticated set of parameterisations of the physical processes in the atmosphere, such as convection and radiation.

Before the addition of chemistry a tracer advection facility was incorporated into the model. This facility allowed passive tracers to be advected in the model using the model derived winds. This "on-line" calculation allows the possibility of incorporating chemically derived fields into the radiation code, for example. The tracer advection code was written in such a way that the maximum number of tracers was limited only by the available computer memory, making it very flexible.

The tracer advection is discretised using the spectral method in the horizontal and the finite difference scheme in the vertical, the same method used for the model's other prognostic variables. However, it is well known that the spectral method suffers from deficiencies in representing fields with large horizontal gradients over few gridpoints $19 \%$. These are often highlighted by the occurrence of negative mixing ratio values and regions of undershoot or overshoot where the minimum or maximum vertical mixing ratio value is exceeded. There are two main causes of these problems. First, the grid associated with a spectral truncation supports more waves than is actually used by the model. Thus, small scale information on the grid can be lost. Second, the Gibbs phenomena can occur when the spectral method is used to represent discontinuities or near discontinuities on the grid. This has important consequences for source regions. Thus, for distributions of species in which sharp gradients form through persistent forcing, the spectral technique may not be the most appropriate, although the converse is true for smooth fields.

\section{THE PHOTOCHEMICAL SCHEME}

The chemistry scheme used in this study is described by Lary /10/. The scheme contains a comprehensive treatment of the gas phase chemistry of $\mathrm{O}_{x}, \mathrm{NO}_{x} \mathrm{ClO}_{x}, \mathrm{HO}_{x}$ and their source gases. It consists of a set of programme modules which have been extensively tested, and validated, as a "stand-alone" diurnal model of the stratosphere. Photolysis rates are calculated using an accurate and efficient photochemical radiative transfer model, which includes the effects of multiple scattering and ground reflection $/ 10,11,12,13 /$.

\section{CHEMISTRY AND DYNAMICS DURING A MAJOR WARMING}

This section briefly presents model results which illustrate the effect of atmospheric dynamics on stratospheric chemistry. The model simulation began with the analysis for 15 Jan 1987 at $12 \mathrm{Z}$ and was integrated forward for 14 days. During the winter of 1986/87 there were a series of warmings, with the peak reached on 19 Jan and the final warming occurring in February. This "explosive" major mid-winter warming was one of the most intense observed (the main features of the stratospheric circulation are described by Naujokat et al. (14/). The model reproduced well the main observational features, including the poleward transport of heat (Figure 1), which led to $10 \mathrm{mb}$ temperatures being
Fig. 1.

The $10 \mathrm{mb}$ SSU temperature field for 19 Jan 1987 (Courtesy of Dr A O'Neill). 
higher at the North Pole than at $60^{\circ} \mathrm{N}$, and the displacement of the polar vortex towards the European side of the hemisphere (Figure 2). These features are of particular importance for the atmospheric chemistry during this period.

Atmospheric dynamics can affect stratospheric chemistry in two main ways, either directly, via a control over the trajectory of air parcels, or indirectly, via its influence over the temperature field. These factors are now illustrated by considering the behaviour of the total column of $\mathrm{NO}_{2}$, and the associated partitioning of reactive nitrogen during the run.

Fig. 2.

The $10 \mathrm{mb}$ SSU geopotential (dam) for 24 Jan 1987 (Courtesy of Dr A O'Neill).

In order to separate the effects of transport and chemistry, Figure 3 shows an $\mathrm{NO}_{2}$ field from a model with no dynamics. Low values are found over the winter pole, and there are sharp gradients in mid-latitudes, similar to the "Noxon cliff" first observed by John Noxon $15,6,7 /$. In this simulation the "Noxon cliff" is formed largely due to the lack of solar illumination present at the winter pole. The cessation of photolysis, for a period of approximately 18 hours leads to a conversion of most of the $\mathrm{NO}_{\mathrm{x}}$ present into the reservoir species $\mathrm{N}_{2} \mathrm{O}_{5}$ and $\mathrm{HNO}_{3}$. During January, the region north of $\approx 70^{\circ} \mathrm{N}$ is in constant darkness, and the cliff is located close to $70^{\circ} \mathrm{N}$, and is symmetrical about the pole.

In integrations with the chemical general circulation model, similar results are found in cases where the stratospheric flow is weak. In this case the symmetry about the pole is a consequence of the relative timescales of dynamical and chemical change of $\mathrm{NO}_{x}\left(=\mathrm{NO}+\mathrm{NO}_{2}\right)$ at $10 \mathrm{mb}$, with chemical changes in the levels of $\mathrm{NO}_{x}$ occurring much more rapidly than dynamical changes. For the "Noxon

N02 (ppbv) - Winter Solstice

Fig. 3. The $\mathrm{NO}_{2}$ distribution at $10 \mathrm{mb}$ for a simulation where only the solar illumination is allowed to change with latitude (temperature and total $\mathrm{NO}_{\mathrm{y}}$ are fixed at mid latitude values). 

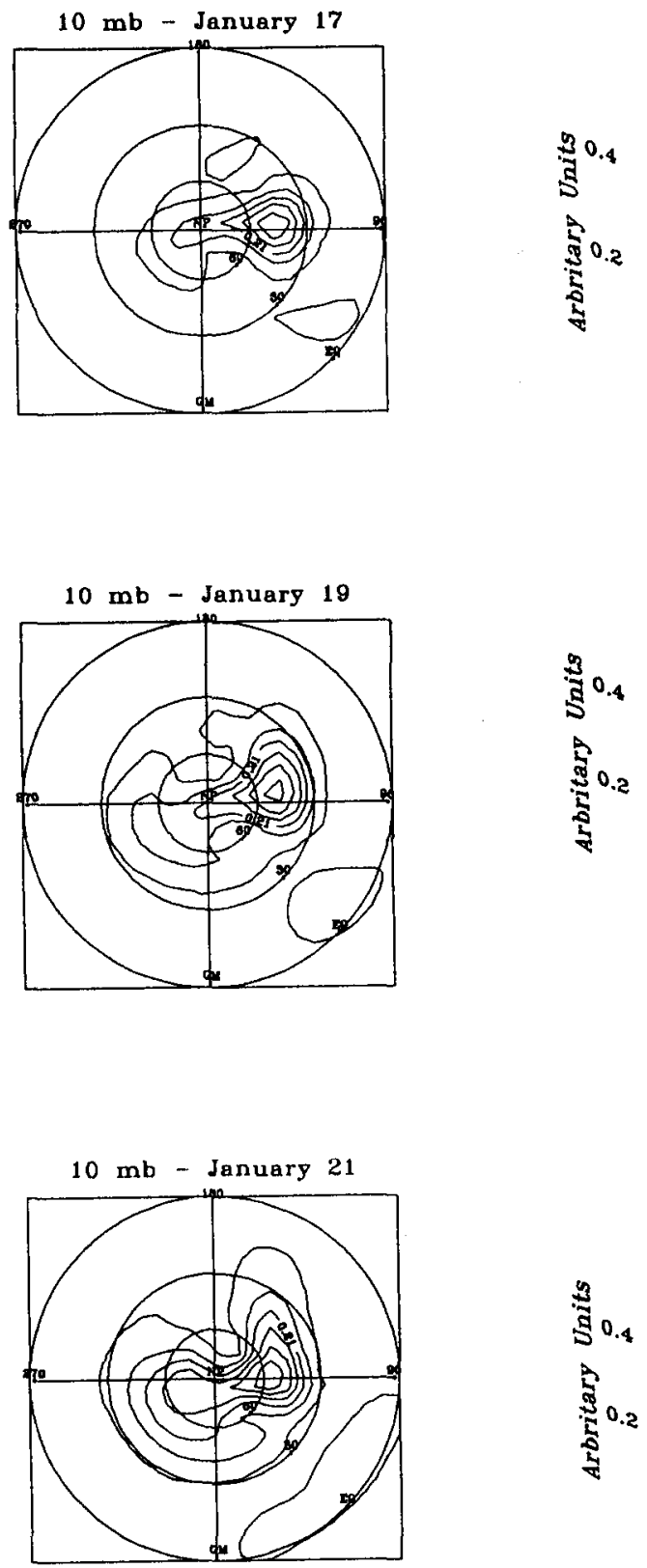

Fig. 4. An inert tracer continually released at $10 \mathrm{mb}$ in a region centred on $50^{\circ} \mathrm{N}, 77.5^{\circ} \mathrm{E}$.

cliff" to be significantly perturbed by atmospheric dynamics, a rapid movement of air must take place over a time period of less than one day. Alternatively, the chemistry must operate on longer time scales, possibly due to the influence of heterogeneous processes. The dynamical perturbation would be most severe when there is a strong flow directly across the winter pole, rapidly moving air which has spent significant periods in darkness to lower latitudes. Such a flow occurred during the major stratospheric warming of January 1987. To illustrate the strength of the cross polar jet at $10 \mathrm{mb}$, an inert tracer was released in the jet entrance region (close to $60^{\circ} \mathrm{N}, 100^{\circ} \mathrm{E}$ at $10 \mathrm{mb}$ ), and in a time period of just two days, significant amounts of air were moved across the pole from 
N02 Column - January 19,1987

Fig. 5. The simulated $\mathrm{NO}_{2}$ Column for $12 \mathrm{Z}$ on 19 Jan $1987\left(10^{15} \mathrm{~mol} . \mathrm{cm}^{-2}\right)$.

$60^{\circ} \mathrm{N}, 90^{\circ} \mathrm{E}$ to $60^{\circ} \mathrm{N}, 270^{\circ} \mathrm{E}$ (Figure 4). The strength of the cross polar jet, which reached a maximum on $19 \mathrm{Jan}$, is reflected in a severe distortion, and elongation towards North America and Greenland, of the region enclosed by the "Noxon cliff" (Figure 5). When the strength of the cross polar jet subsided later in the month, the region enclosed by the "Noxon cliff" became more symmetrical, and was again centred over the pole.

The temperature and ozone field also have an important influence over the partitioning of reactive nitrogen species adopted during a given event. For example, during a stratospheric warming, the highest temperatures are associated with the rapid air flow at the peak of the cross polar jet. The fraction of reactive nitrogen present as $\mathrm{HNO}_{3}$ or $\mathrm{N}_{2} \mathrm{O}_{5}$ is a function of temperature. With the high

N205 (ppbv) - January 19,1987

Fig. 6. The simulated $\mathrm{N}_{2} \mathrm{O}_{5}$ distribution at $10 \mathrm{mb}$ for $12 \mathrm{Z}, 19 \mathrm{Jan}$ (ppbv). 
temperatures and high ozone concentrations found close to the pole on 19 Jan the levels of $\mathrm{N}_{2} \mathrm{O}_{5}$ are high (Figure 6) and accordingly the levels of $\mathrm{HNO}_{3}$ present at $10 \mathrm{mb}$ are significantly reduced.

\section{CONCLUSIONS}

Results have been presented from an integration of a three-dimensional spectral model including a detailed description of gas-phase atmospheric chemistry. The integration spanned the period of a major stratospheric warming in January 1987. Although not shown here, modelled ozone fields agreed reasonably well with available measurements. The $\mathrm{NO}_{2}$ column field shows a feature very similar to that reported by Noxon $/ 5,6,7 /$ with low column amounts in high latitudes. This has been shown to be due to conversion during darkness to $\mathrm{N}_{2} \mathrm{O}_{5}$. High polar temperatures and high polar ozone concentrations, produced during the sudden warming, both favour the gasphase production of $\mathrm{N}_{2} \mathrm{O}_{5}$. During the most disturbed phase of the warming the low $\mathrm{NO}_{2}$ values are advected significantly southwards. It will be the aim of subsequent studies to investigate the role of heterogeneous processes in the production of the "Noxon cliff" and in particular to see whether the conversion of $\mathrm{N}_{2} \mathrm{O}_{5}$ to $\mathrm{HNO}_{3}$ on ice surfaces is necessary to reproduce the observations of the "Noxon cliff" in middle latitudes. It is hoped that future studies will be made with the extended general circulation model which extends to higher altitudes (Gray et al. this issue).

\section{ACKNOWLEDGEMENTS}

It is a pleasure to acknowledge our many UGAMP colleagues, too numerous to mention by name. Special thanks must be given to the UGAMP core group at Reading University, particularly Mike Blackburn and John Thuburn. We are indebted at Cambridge to work done by Stephen Beagley. David Lary thanks SERC for a studentship, Glenn Carver is a member of the UGAMP Central Unit, funded by NERC. Without the kind assistance of ECMWF this study would not have been possible. David Dent of ECMWF is thanked for a number of helpful conversations.

\section{REFERENCES}

1. J.D. Mahlman, and W.J. Moxim, Tracer simulation using a global general circulation model: Results from mid-latitude instantaneous source experiment, J. Atmos. Sci., 41, 2029-2051, 1978

2. J.A. Kaye, and R.B. Rood, Chemistry and transport in a three-dimensional stratospheric model: Chlorine species during a simulated stratospheric warming, J. Geophys. Res., 94, D1, 1057-1083, 1989

3. W.L. Grose, J.E. Nealy, R.E. Turner, W.T. Blackshear, Modelling the transport of chemically active constituents in the stratosphere, in Transport processes in the middle atmosphere, 229-250, D. Reidel, Hingham, Mass. 1987

4. D. Cariolle, and M. Deque, Southern Hemisphere medium-scale waves and total ozone disturbances in a spectral general circulation model, J. Geophys. Res., 91, 10,825-10,846, 1986

5. J.F. Noxon, E.C. Whipple Jr, and R.S. Hyde, Stratospheric $\mathrm{NO}_{2}, 1$. Observational method and behaviour at mid-latitude, J. Geophys. Res., 84, 5047-5065, 1979a.

6. J.F. Noxon, W.R. Henderson, and R.B. Norton, Stratospheric $\mathrm{NO}_{2}, 3$. The effects of large scale horizontal transport, J. Geophys. Res., 88, 5240-5248, 1983. 
7. J. F. Noxon, W. R. Henderson, and R. B. Norton, Stratospheric $\mathrm{NO}_{2}, 3$. The effects of large scale horizontal transport, J. Geophys. Res., 88, 5240-5248, 1983.

8. A.J. Simmons, D.M. Burridge, An energy and angular momentum conserving vertical finite difference scheme and hybrid vertical coordinates. Mon. Wea. Rev., 109, 758-766, 1981.

9. R.B. Rood, Numerical advection algorithms and their role in atmospheric transport and chemistry models, Rev. Geophys., 25, 71-100, 1987.

10. D.J. Lary, Photochemical studies with a three-dimensional model of the atmosphere, $P h D$ Thesis, University of Cambridge, 1991.

11. R.R. Meier, D.E. Anderson Jr, and M. Nicolet, Radiation field in the troposphere and stratosphere from 240-1000nm-I. General Analysis, Planet. Space Sci,30, 923-933, 1982.

12. M. Nicolet, R.R. Meier, and D.E. Anderson Jr, Radiation field in the troposphere and stratosphere from 240-1000nm-II. Numerical Analysis, Planet. Space Sci.,30, 935-983, 1982.

13. D.E. Anderson Jr. The troposphere-stratosphere radiation field at twilight: A spherical model, Planet. Space Sci,12, 1517-1523, 1983.

14. B. Naujokat, K. Labitzke, R. Lenschow, K. Petzoldt, R.C. Wohlfart, The stratospheric winter 1986/87: A major warming 35 years after they were first detected, Beilage zur Berliner Wetterkarte, 56/87 SO 9/87, 1987. 